\title{
The pervasive role of microRNAs in arrhythmia: Animal models and novel discoveries
}

\author{
Guzzolino E and Pitto L* \\ Institute of Clinical Physiology, National Research Council, Pisa, Italy
}

\section{Introduction}

Cardiac arrhythmia and in particular atrial fibrillation (AF), which is the most common sustained arrhythmia, is one of the main cause of morbidity and mortality worldwide [1] The aetiology is attributable to multiple factors such as genetic, lifestyle and other pathological stress [2]. For pathologies as long QT syndrome (LQTS), Brugada syndrome, Timothy syndrome, "torsade de pointes" phenotype and cardiac sudden death, a genetic aetiology was recognized. However, even for arrhythmia syndromes with genetic basis, prediction of the phenotypic expression was not simple because of the extreme variability in the severity of disease manifestation also among family members presenting the same mutation [3]. Indeed, the arrhythmic phenotype has features of a "complex phenotype" which probably reflects the intricate and interconnected events that occur in cardiac cells, from the action potential activation to the regulation of excitation contraction coupling event. For these reasons, cardiac arrhythmia remains a big challenge for modern drug treatment and the comprehension of how DNA variation and other concomitant factors might influence disease is of great importance. In this panorama, microRNAs (miRNAs), small molecules of 22-25 nucleotides negatively regulating gene expression [4] might represent an interesting field of investigation. Although they are no longer a new discovery, the interest in their functions in heart pathologies and in their possible use as therapeutic molecular drugs, continues to be under study, thanks also to their unique capacity to contemporarily modulate more genes and whole pathways. Following this prospective, several clinical trials have been initiated using miRNAand siRNA-based therapeutics [5]. Even if we are far to create a miRNAbased treatment in arrhythmia, different evidences demonstrated the possibility to pursue this aim for cardiac pathologies as suggested by the action of 2 molecular drugs MGN-9103 [6] and MGN-1374 [7] developed by an American company, used to treat heart failure and cardiac infarction respectively.

A deeper knowledge of roles played by miRNAs in the development of cardiac arrhythmias is a crucial prerequisite to enable the use of these small molecules as new drugs or drug targets. Although in the literature is present an extensive characterization of miRNAs targeting genes involved in heart physiology, there are few papers describing miRNAs with a causative role in arrhythmogenesis. This review is focused on miRNA genes deeply involved in arrhythmia and on the principal animal models, which allowed to decipher the functional miRNA role in arrhythmic cardiac pathologies.

\section{Genome wide analyses and miRNAs}

The advent of genome wide approach allowed to discover association among genetic loci and pathological states and represents the classic genetic analyses to find signature for heritable risk factors.

During last years the attention focused on mutation located in coding sequence (CDS) of genes [3]. A genome wide analyses unveiled a strong association between two sequence variants on chromosome $4 \mathrm{q} 25$ and AF. Both variants were in close proximity to PITX2 gene [8] and this observation leads to discover a direct regulation of miR-17-92 and miR106b-25 clusters [9] by PITX2 and successively the involvement of these miRNA clusters in arrhythmia.

Besides "indirect" approaches to identify miRNAs with a role in arrhythmia, the number of studies focused on discovering human miRNA sequence variants are rather limited. In the analyses of Hedley et al. DNA sequence variations in genes codifying for miR-1 and miR133a in LQT probands were inspected, but the results showed that these miRNA variants were not correlated to LQTS in this cohort [10]. On the contrary, a sequencing of 2600 individual DNAs allowed to find a naturally occurring mutation in the mir-499. Overexpression of wildtype and mutant miR-499 in murine heart showed that the presence of the miR-499 c17 mutation alters myocardial protein levels of the miR-499 targets leading to a less severe cardiac remodelling after heart failure [11].

Sequencing the regulatory regions of genes, not only the CDS, should be of particular importance in miRNA investigation. Indeed, functional microRNAs bind the mRNA targets through RNA-RNA base pairing generally in the 3'-UTR. The canonical recognition requires usually a perfect pairing between roughly a 6-8 nucleotide stretch of the $5^{\prime}$-end of the miRNA, known as "seed" $[4,12,13]$ and the corresponding complementary sequence in the target 3'-UTR, called "seed-match".

Therefore, mutations in miRNA seed-match outside the CDS of genes related to arrhythmic pathologies, might be the causative effect in cases of arrhythmias with yet-unknown genetic aetiology. The literature describes several examples of polymorphisms in the 3'-UTR of key genes associated to heart diseases corroborating this hypothesis [14-19]. However as mentioned before, these analyses are mainly association studies which do not investigate the functional meaning of these variants. Insightful was the work of Hoffman et al. focused on the analysis of SHOX2 in AF patients. The authors showed in particular that patients carrying the $3^{\prime} \mathrm{UTR}$ variant c. ${ }^{\star} 28 \mathrm{~T}>\mathrm{C}$ present significantly longer PR intervals. Mechanistically, the variant creates a new functional

${ }^{\star}$ Correspondence to: Letizia Pitto, Institute of Clinical Physiology, National Research Council, Pisa, Italy, E-mail: 1.pitto@ifc.cnr.it

Received: November 22, 2018; Accepted: December 07, 2018; Published: December 12, 2018 
binding site for hsa-miR-92b-5p, which may reduce SHOX2 expression and thereby facilitate proarrhythmogenic remodelling leading to $\mathrm{AF}$ [20].

MiRNA effectiveness is based on thermodynamic forces and affinity to targets, which are parameters used in several algorithms to predict RNA-RNA recognition. Changes in this affinity affects the balance of gene expression and the contribution of each miRNA in regulating shared mRNA targets or the possibility for a miRNA to interact with competitors in a big net of communication [21,22]. The amount of functional miRNA results from the balance among competitive targets and the final result is a combination of RNA abundance and phenomena of redundancy with palliative action. In this very complex panorama, emerged long non-coding RNA [lncRNA] as new players in arrhythmic disease, because they can behave as miRNA sponges. Evidences of their possible role emerged from the observation of a different expression profiles of lncRNA in AF patients [23]. An example is the lncRNA Kcna2 antisense RNA [24] whose expression increases in rats with congestive heart failure $[\mathrm{CHF}]$ and that has been recently shown to contribute to ventricular arrhythmia via silencing Kcna2. Another interesting case of direct relation among miRNAs and lncRNAs in arrhythmic pathologies is the lncRNA TCONS_00075467 that modulates atrial electrical remodelling by sponging miR-328 which regulates the downstream CACNA1C protein [25]. Moreover, a complex molecular mechanism was discovered by Zhu et al. for LncRNA MALAT1 that was found upregulated in cardiomyocytes from arrhythmic model rats [26]. It was suggested that MALAT1 might act as a competing endogenous RNA for miR-200c that targets the high-mobility group box 1 (HMGB1) protein, a highly conserved nuclear protein that operates as a chromatin-binding factor. The upregulation of HMGB consequent to the downregulation of miR-200 might mediate the downregulation of the transient outward potassium current [26].

\section{The interplay between miRNAs and Transcription Factors}

Since miRNAs and transcription factors (TFs) are trans-acting factors that interact with cis-regulatory elements, they potentially generate a complex combinatorial code. This is clearly shown by the observation that genes with many TF binding sites have a higher probability to be controlled by miRNAs than genes with few TF binding sites [27]. Emerging evidences indicate a crucial miRNA/ TF interplay in many biological processes and in arrhythmia. PITX2, TBX5 and MYOCD are among the TFs principally involved in heart morphogenesis and especially studied for AF [28]. Classic analysis, based on in silico approach and in vitro assays to determine miRNA/ mRNA functional interaction, revealed miR-21, miR-10b, and miR-1 as mayor regulator of PITX2C, TBX5, and MYOCD respectively in AF patients [29]. A more sophisticated molecular screening method, the Par-Clip method, found several other miRNAs targeting PITX2 such as miR-153, miR-5692, miR-369, miR-4517, miR-5583, miR-377, miR-374 [30] whose role in pathogenesis is still unknown.

By high throughput comparison of zebrafish embryos depleted or not for Tbx5 [31] the miR-17-92 cluster was identified as directly regulated by Tbx 5 . In zebrafish, cardiac defects generated by Tbx 5 depletion can be partially reverted by modulation of miR-19a, a member of this cluster, in line with a role of this miRNA as Tbx 5 functional effector. Furthermore, miR-19a is able to affect heart rate control in HoltOram syndrome [HOS] fish by targeting the Sphingosine-1-Phosphate Receptor 1 which is highly expressed in mammalian cardiomyocytes and implicated in numerous cardiovascular processes [32]. Moreover
miR-17-92 cluster has been also shown to directly repress genes, such as SHOX2 and TBX3, that are required for sinoatrial node development while miR-1 also targets the zinc finger homeobox 3 gene (ZFHX3) which is another important AF susceptibility-conferring gene [33-35].

A potentially interesting factor that, differently from the previous we mentioned, is considered as transcriptional repressor, is the neuronrestrictive silencer factor (NRSF) [36], that selectively regulates the expression of multiple fetal cardiac genes [37]. In transgenic mice the expression of a NRSF dominant-negative mutant (dnNRSF) leads to cardiomyopathy, high susceptibility to arrhythmias and sudden death [38]. Although a direct relationship between NRSF gene and regulating miRNAs is not still discovered in arrhythmias, intriguingly, a PAR-clip extensive analysis of miRNA-RNA-targets interaction revealed miR106 and miR-17 [39], known to be involved in arrhythmias [see next chapter], among the miRNAs able to recognize NRSF.

\section{Functional characterization of miRNAs in animal models of arrhythmia}

The "OMIC" studies, which are fascinating and nowadays indispensable for genetic investigation, can be tricky in the phases of matching and interpretation for miRNA data analysis. Slight modification in miRNA expression might lead to a pathological state and methods used to evaluate miRNA abundance in a sample might be critic, with a risk to lose information. An example of a big effort ended in a poor result was a study of 2016 aimed to find a strong net of regulation among deregulated miRNAs and related validated targets in AF [40]. From this study based on microarray expression profiles and bioinformatics analysis, emerged only generic classes of gene ontology on "metabolism" and "regulation of cellular/biological process" which does not improve our knowledge about the regulatory network in AF.

Significant steps forward in the characterization of miRNAs causative of arrhythmias have been done by exploiting transgenic/ mutant animal models, which can also allow to evaluate miRNA therapeutic potential.

The miR-1, maybe the most cited cardiac miRNA, is highly expressed in cardiac tissue and found deregulated in the major part of heart pathology studies. MiR-1 is involved both in cardiogenesis and cardiac conduction system development [41]. A recent study based on transgenic mice with cardiomyocytes-specific over-expression of miRNA-1, revealed a new contribution in arrhythmias. This study showed that miR-1 overexpression dysregulates intracellular trafficking and, as a consequence, alters calcium handling by targeting Syntaxin 6, which indirectly decreased Cav1.2 expression [42]. Due to the huge amount of miR-1 target genes in cardiac cells [43], the use of this miRNA, or of similar highly multitasking miRNAs, in gene therapy is challenging. A potential therapeutic role of miR-1 is shown only in models of myocardial infarction [44] and cardiac reprogramming [45].

The miR-17-92 cluster is known to be involved in heart development and its deregulation was associated to pathological cardiac states, but the physiological involvement of each cluster member is not completely understood and is still under study. MiR-19a/b members of the cluster were associated to arrhythmias because they target cx43 gene [46]. Exploiting the simplified but conserved zebrafish model, the involvement of miR-19 in heart rate was uncovered. Zebrafish lacking miR-19b by CRISPR-mediated knockout show severe bradycardia as well as susceptibility to arrhythmias and cardiomyopathy. Moreover miR-19b affects action potential duration [APD], in part, by directly targeting KCNE4 [47]. 
Table 1. A short list of validated miRNA-mRNA targets derived from literature analysis published from 2016 to 2018 and generated by searching for genes known to be involved in arrhythmia or for new discoveries underlie arrhythmic disorders

\begin{tabular}{|c|c|c|c|c|}
\hline miRNA & Target gene & Specie & Model & Note \\
\hline miR-135b & CACNA1C & mouse & hypertrophy & 59 \\
\hline $\operatorname{miR}-34 \mathrm{a}$ & Ank-B & Human & $\mathrm{AF}$ & 60 \\
\hline miR-29a & CACNA1C & Human and mouse & $\mathrm{AF}$ and in vitro HL1 & 61 \\
\hline $\operatorname{miR}-223$ & KCND2 & Rat & myocardial infarction & 62 \\
\hline miR-1231 & CACNA2D2 & Human and rat & myocardial infarction & 63 \\
\hline $\operatorname{miR}-132$ & CTGF & Human and dog & $\begin{array}{l}\text { AF-dependent structural } \\
\text { remodelling }\end{array}$ & 63 \\
\hline $\mathrm{miR}-34 \mathrm{~b} / \mathrm{c}$ & $\begin{array}{l}\text { SCN5A } \\
\text { SCN1B }\end{array}$ & Human and rat & icells & 64 \\
\hline miR-130a & $\mathrm{DSC} 2$ & mouse & Arrhythmogenic Cardiomyopathy & 65 \\
\hline $\operatorname{miR}-31$ & $\begin{array}{l}\text { nNOS } \\
\text { DYS }\end{array}$ & Human and mouse & $\mathrm{AF}$ & 66 \\
\hline
\end{tabular}

To gain insight into miR-17-92 cluster involvement in arrhythmia, miR-17-92 $2^{\text {null/+ }}$ mice were studied since miR-17-92 ${ }^{\text {null/null }}$ mutants show post-natal lethality. This study included also miR-106b-25 cluster that shears with miR-17-92 cluster miRNAs belonging to the same family and that, together to miR-17-92 cluster, is controlled by PITX2 [see also previous chapter of this review]. Although neither miR-17-92 $2^{\text {null/+}}$ nor miR-106b-25 $5^{\text {null/++}}$ or miR-106b-25 $5^{\text {null/null }}$ mice show spontaneous episodes of AF, in condition of pacing-induced AF, the loss of miR17-92 and miR-106b-25 increases susceptibility to AF. Interestingly, mice with miR-17-92 cardiac-specific knockout or miR-106b-25 haploinsufficiency showed arrhythmogenic events and senoatrial node dysfunction [9]. In a different study, miR-106b-25 $5^{\text {null/null }}$ mice exhibited enhanced sarcoplasmic reticulum $\mathrm{Ca}^{2+}$-leak and increased ryanodine receptor type-2 [RyR2] expression [34] in line with studies showing that the level of RyR2 protein was elevated in atria of paroxysmal AF [pAF] patients [48].

In a different manner, cardiac specific overexpression of miR-206 in adult mice was shown to suppress $\mathrm{cx} 43$ expression and to induce abnormal heart rate and PR interval causing also shortening of lifespan in the experimental mice [49]. Moreover, upregulation of miR206 cluster induced by overexpression of HMGB1, was shown to induce downregulation of TimP3, an inhibitor of matrix metalloproteinases 2 and 9 [MMP-2 and MMP-9] [50,51]. This data suggests that miR206 modulation, affecting collagen deposition activity and enhancing collagenolytic activity, can potentially have not only a causative role in arrhythmogenesis but also impact AF remodelling phenomena. Another miRNA potentially involved in AF remodelling is miR328 that we already mentioned because controlled by the lncRNA TCONS_00075467. This miRNA was identified in a miRNA profiling performed on a canine model of AF induced by atrial tachypacing and it resulted also overexpressed in cardiac tissue of AF patients. MiR-328 cardiac specific overexpression in mice [25] recapitulated the phenotypes of AF, increased AF vulnerability, diminished L-type $\mathrm{Ca}^{2+}$ current, and shortened atrial action potential while genetic knockdown of endogenous miR-328 reduced AF susceptibility.

A further miRNA which is deregulated in AF patients is miR-208 [52], considered a cardiac specific miRNA. Transgenic overexpression of miR-208a in the heart affects both cardiac growth and conduction. On one side miR-208 targets thyroid hormone-associated protein 1 and myostatin, which are negative regulators of muscle growth and hypertrophy and, on the other side, this miRNA affects the cardiac conduction system by controlling GATA4 which in turn regulates the expression of the cardiac TFs homeodomain-only protein [Hop] and the gap junction protein $\mathrm{cx} 40$ [53].
Deregulation of miR-499 and miR-27b have been also shown to increase AF vulnerability although studies in animal model clearly demonstrated that these miRNAs are not strictly arrhythmogenic. MiR-499 is overexpressed in patients with permanent AF [54] and its constitutive overexpression in transgenic murine hearts caused hypertrophy. MiR-499 deregulation seems to primarily influence cardiac gene expression and predispose to cardiac stress-induced dysfunction [55]. MiR-27b, identified because up-regulated in a mouse model of high fat diet [56], increases vulnerability to atrial arrhythmia by targeting cx43. Transgenic mice with cardio-specific overexpression of miR-27b born normally and have normal cardiac structure, although $30 \%$ of them develop significant cardiac hypertrophy at 3 months of age and die suddenly between 6 and 12 months of age [57].

A recent miRNA revealed to play a possible important role in arrhythmia is miR-182 identified in mouse model of HOS as downregulated by Tbx5. This microRNA has been functionally studied in zebrafish where its role in cardiac development and function has been confirmed. Upregulation of miR-182 induces downregulation of several calcium channel proteins and alteration of calcium handling. In line with this observation, stable overexpression of miR-182 in zebrafish myocardium is able to induce spontaneous events of arrhythmia overtime [58].

\section{New miRNAs discovered targeting gene involved in Arrhythmia}

Besides the microRNAs just described, the following table presents a short list of validated miRNA-mRNA targets derived from literature analysis published from 2016 to 2018 and generated by searching for genes known to be involved in arrhythmia or for new discoveries underlie arrhythmic disorders (Table 1).

\section{References}

1. Wakili R, Voigt N, Kaab S, Dobrev D, Nattel S (2011) Recent advances in the molecular pathophysiology of atrial fibrillation. J Clin Invest 121: 2955-2968. [Crossref]

2. Schoonderwoerd BA, Smit MD, Pen L, Van Gelder IC (2008) New risk factors for atrial fibrillation: causes of 'not-so-lone atrial fibrillation'. Europace 10: 668-673. [Crossref]

3. Wilde AA, Bezzina CR (2005) Genetics of cardiac arrhythmias. Heart 91: 1352-1358. [Crossref]

4. Bartel DP (2009) MicroRNAs: target recognition and regulatory functions. Cell 136: 215-233. [Crossref]

5. Chakraborty C, Sharma AR, Sharma G, Doss CGP, Lee SS (2017) Therapeutic miRNA and siRNA: Moving from Bench to Clinic as Next Generation Medicine. Molecular therapy Nucleic acids 8: 132-143. [Crossref]

6. Montgomery RL, Hullinger TG, Semus HM, Dickinson BA, Seto AG, et al. (2011) Therapeutic inhibition of miR-208a improves cardiac function and survival during heart failure. Circulation 124: 1537-1547. [Crossref] 
7. Hullinger TG, Montgomery RL, Seto AG, Dickinson BA, Semus HM, et al. (2012) Inhibition of miR-15 protects against cardiac ischemic injury. Circ Res 110: 71-81. [Crossref]

8. Gudbjartsson DF, Arnar DO, Helgadottir A, Gretarsdottir S, Holm H, et al. (2007) Variants conferring risk of atrial fibrillation on chromosome 4q25. Nature 448: 353 357. [Crossref]

9. Wang J, Bai Y, Li N, Ye W, Zhang M, et al. (2014) Pitx2-microRNA pathway that delimits sinoatrial node development and inhibits predisposition to atrial fibrillation. Proc Natl Acad Sci U S A 111: 9181-6. [Crossref]

10. Hedley PL, Carlsen AL, Christiansen KM, Kanters JK, Behr ER, et al. (2014) MicroRNAs in cardiac arrhythmia: DNA sequence variation of MiR-1 and MiR-133A in long QT syndrome. Scand J Clin Lab Invest 74: 485-491. [Crossref]

11. Dorn GW 2nd, Matkovich SJ, Eschenbacher WH, Zhang Y (2012) A human 3' miR499 mutation alters cardiac mRNA targeting and function. Circulation research 110: 958-67. [Crossref]

12. Lewis BP, Shih IH, Jones-Rhoades MW, Bartel DP, Burge CB (2003) Prediction of mammalian microRNA targets. Cell 115: 787-798. [Crossref]

13. Brennecke J, Stark A, Russell RB, Cohen SM (2005) Principles of microRNA-target recognition. PLoS biology 3: e85.

14. Wang F, Liu D, Zhang RR, Yu LW, Zhao JY, et al. (2017) A TBX5 3'UTR variant increases the risk of congenital heart disease in the Han Chinese population. Cell discovery 3: 17026 .

15. Wang J, Zhang RR, Cai K, Yang Q, Duan WY, et al. (2018) Susceptibility to congenital heart defects associated with a polymorphism in TBX2 3' untranslated region in the Han Chinese population. Pediatric research 18. [Crossref]

16. Chu HM, Feng MJ, Li YG, Zhang YX, Ma JF, et al. (2013) Polymorphisms but not mutations of the KCNQ1 gene are associated with lone atrial fibrillation in the Chinese Han population. The Scientific World Journal 373454

17. Paludan-Muller C, Svendsen JH, Olesen MS (2016) The role of common genetic variants in atrial fibrillation. J Electrocardiol 49: 864-870. [Crossref]

18. Ren X, Xu C, Zhan C, Yang Y, Shi L, et al. (2010) Identification of NPPA variants associated with atrial fibrillation in a Chinese GeneID population. Clinica chimica acto 411: 481-485. [Crossref]

19. Tian L, Liu G, Wang L, Zheng M, Li Y (2015) KCNA5 gene polymorphism associate with idiopathic atrial fibrillation. Int J Clin Exp Med 8: 9890-9896. [Crossref]

20. Hoffmann S, Clauss S, Berger IM (2016) Coding and non-coding variants in the SHOX2 gene in patients with early-onset atrial fibrillation. Basic Res Cardiol 111: 36. [Crossref]

21. Bosson AD, Zamudio JR, Sharp PA (2014) Endogenous miRNA and target concentrations determine susceptibility to potential ceRNA competition. Molecular cell 56: 347-359.

22. Denzler R, Agarwal V, Stefano J, Bartel DP, Stoffel M (2014) Assessing the ceRNA hypothesis with quantitative measurements of miRNA and target abundance. Mol Cell 54: 766-776. [Crossref]

23. Ruan Z, Sun X, Sheng H, Zhu L (2015) Long non-coding RNA expression profile in atrial fibrillation. International journal of clinical and experimental pathology 8: $8402-8410$.

24. Long QQ, Wang H, Gao W, Fan Y, Li YF (2017) Long Noncoding RNA Kcna2 Antisense RNA Contributes to Ventricular Arrhythmias via Silencing Kcna2 in Rats With Congestive Heart Failure. Journal of the American Heart Association 6.

25. Lu Y, Zhang Y, Wang N, Pan Z, Gao X, et al. (2010) MicroRNA-328 contributes to adverse electrical remodeling in atrial fibrillation. Circulation 122: 2378-2387.

26. Zhu P, Yang M, Ren H, Shen G, Chen J, et al. (2018) Long noncoding RNA MALAT1 downregulates cardiac transient outward potassium current by regulating miR-200c/ HMGB1 pathway. Journal of cellular biochemistry 119: 10239-10249.

27. Cui Q, Yu Z, Pan Y, Purisima EO, Wang E (2007) MicroRNAs preferentially target the genes with high transcriptional regulation complexity. Biochemical and biophysical research communications $352:$ 733-738.

28. Mahida S (2014) Transcription factors and atrial fibrillation. Cardiovascular Research 101: 194-202. [Crossref]

29. Torrado M, Franco D, Lozano-Velasco E, Hernandez-Torres F, Calvino R, et al. (2015) A MicroRNA-Transcription Factor Blueprint for Early Atrial Arrhythmogenic Remodeling. BioMed research international 263151.
30. Kishore S, Jaskiewicz L, Burger L, Hausser J, Khorshid M, et al. (2011) A quantitative analysis of CLIP methods for identifying binding sites of RNA-binding proteins. Nature methods 8: 559-564.

31. Chiavacci E, D'Aurizio R, Guzzolino E, Russo F, Baumgart M, et al. (2015) MicroRNA 19a replacement partially rescues fin and cardiac defects in zebrafish model of Holt Oram syndrome. Scientific reports 5: 18240.

32. Guzzolino E, Chiavacci E, Ahuja N, Mariani L, Evangelista M, et al. (2018) Posttranscriptional Modulation of Sphingosine-1-Phosphate Receptor 1 by miR-19a Affects Cardiovascular Development in Zebrafish. Frontiers in cell and developmental biology 6: 58 .

33. Zhai C, Cong H, Liu Y, Zhang Y, Liu X, et al. (2015) Rs 7193343 polymorphism in zinc finger homeobox 3 (ZFHX3) gene and atrial fibrillation: an updated meta-analysis of 10 case-control comparisons. BMC cardiovascular disorders 15: 58. [Crossref]

34. Benjamin EJ, Rice KM, Arking DE, Pfeufer A, van Noord C, et al. (2009) Variants in ZFHX3 are associated with atrial fibrillation in individuals of European ancestry. Nature genetics 41: 879-881.

35. Liu Y, Ni B, Lin Y, Chen XG, Fang Z, et al. (2014) Genetic polymorphisms in ZFHX3 are associated with atrial fibrillation in a Chinese Han population. PLoS One 9: e101318. [Crossref]

36. Yu J, Vodyanik MA, Smuga-Otto K, Antosiewicz-Bourget J, Frane JL, et al. (2007) Induced pluripotent stem cell lines derived from human somatic cells. Science 318: 1917-1920. [Crossref]

37. Kuwahara K, Saito Y, Takano M, Arai Y, Yasuno S, et al. (2003) NRSF regulates the fetal cardiac gene program and maintains normal cardiac structure and function. The EMBO journal 22: 6310-6321.

38. Cerbai E, Sartiani L, DePaoli P, Pino R, Maccherini M, et al. (2001) The properties of the pacemaker current $\mathrm{I}(\mathrm{F})$ in human ventricular myocytes are modulated by cardiac disease. J Mol Cell Cardiol 33: 441-448. [Crossref]

39. Memczak S, Jens M, Elefsinioti A, Torti F, Krueger J, et al. (2013) Circular RNAs are a large class of animal RNAs with regulatory potency. Nature 495: 333-338. [Crossref]

40. Wang T, Wang B (2016) Identification of microRNA-mRNA interactions in atria fibrillation using microarray expression profiles and bioinformatics analysis. Molecular medicine reports 13: 4535-4540.

41. Zhao Y, Ransom JF, Li A, Vedantham V, von Drehle M, et al. (2007) Dysregulation of cardiogenesis, cardiac conduction, and cell cycle in mice lacking miRNA-1-2. Cell 129: 303-317. [Crossref]

42. Su X, Liang H, Wang H, Chen G, Jiang H, et al. (2017) Over-expression of microRNA-1 causes arrhythmia by disturbing intracellular trafficking system. Scientific Reports 7 46259 .

43. Shan H, Li X, Pan Z, Zhang L, Cai B, et al. (2009) Tanshinone IIA protects agains sudden cardiac death induced by lethal arrhythmias via repression of microRNA-1. British journal of pharmacology 158: 1227-1235.

44. Liao C, Gui Y, Guo Y, Xu D (2016) The regulatory function of microRNA-1 in arrhythmias. Mol Biosyst 12: 328-333. [Crossref]

45. Jayawardena TM, Egemnazarov B, Finch EA, Zhang L, Payne JA, et al. (2012) MicroRNA-mediated in vitro and in vivo direct reprogramming of cardiac fibroblasts to cardiomyocytes. Circ Res 110: 1465-1473. [Crossref]

46. Danielson LS, Park DS, Rotllan N, Chamorro-Jorganes A, Guijarro MV, et al (2013) Cardiovascular dysregulation of miR-17-92 causes a lethal hypertrophic cardiomyopathy and arrhythmogenesis. FASEB Journal 27: 1460-1467. [Crossref]

47. Benz A, Kossack M, Auth D, Seyler C, Zitron E, et al. (2016) miR-19b Regulates Ventricular Action Potential Duration in Zebrafish. Scientific Reports 6: 36033.

48. Chiang DY, Kongchan N, Beavers DL, Alsina KM, Voigt N, et al. (2014) Loss of microRNA-106b-25 cluster promotes atrial fibrillation by enhancing ryanodine receptor type-2 expression and calcium release. Circulation Arrhythmia and electrophysiology 7: 1214-1222. [Crossref]

49. Jin Y, Zhou TY, Cao JN, Feng QT, Fu YJ, et al. (2018) MicroRNA-206 Downregulates Connexin43 in Cardiomyocytes to Induce Cardiac Arrhythmias in a Transgenic Mouse Model. Heart, lung \& circulation 4. [Crossref]

50. Limana F, Esposito G, D'Arcangelo D, Di Carlo A, Romani S, et al. (2011) HMGB1 attenuates cardiac remodelling in the failing heart via enhanced cardiac regeneration and miR-206-mediated inhibition of TIMP-3. PloS one 6: e19845.

51. Shan ZX, Lin QX, Fu YH, Deng CY, Zhou ZL, et al. (2009) Upregulated expression of miR-1/miR-206 in a rat model of myocardial infarction. Biochemical and biophysical research communications 381:597-601. 
52. Canon S, Caballero R, Herraiz-Martinez A, Perez-Hernandez M, Lopez B, et al. (2016) miR-208b upregulation interferes with calcium handling in HL-1 atrial myocytes: Implications in human chronic atrial fibrillation. $J$ Mol Cell Cardiol 99: 162-173. [Crossref]

53. Callis TE, Pandya K, Seok HY, Tang RH, Tatsuguchi M, et al. (2009) MicroRNA-208a is a regulator of cardiac hypertrophy and conduction in mice. J Clin Invest 119: 27722786. [Crossref]

54. Ling TY, Wang XL, Chai Q, Lau TW, Koestler CM, et al. (2013) Regulation of the SK3 channel by microRNA-499--potential role in atrial fibrillation. Heart Rhythm 10: 1001-1009. [Crossref]

55. Shieh JT, Huang Y, Gilmore J, Srivastava D (2011) Elevated miR-499 levels blunt the cardiac stress response. PLoS One 6: e19481. [Crossref]

56. Takahashi K, Sasano T, Sugiyama K, Kurokawa J, Tamura N, et al. (2016) High-fat diet increases vulnerability to atrial arrhythmia by conduction disturbance via miR-27b. Journal of molecular and cellular cardiology 90: 38-46.

57. Wang J, Song Y, Zhang Y, Xiao H, Sun Q, et al. (2012) Cardiomyocyte overexpression of miR-27b induces cardiac hypertrophy and dysfunction in mice. Cell research 22 : 516-527.

58. Guzzolino E, Ahuja N, Garrity D, Pellegrino M, Mercatanti A, et al. (2018) 229Stable and transient miR-182 overexpression reproduces morphological and physiological cardiac defects caused by Tbx5 depletion in zebrafish. Cardiovascular Research 114: S59-S59.
59. Chu Q, Li A, Chen X, Qin Y, Sun X, et al. (2018) Overexpression of miR-135b attenuates pathological cardiac hypertrophy by targeting CACNA1C. International journal of cardiology 269: 235-241. [Crossref]

60. Zhu Y, Feng Z, Cheng W, Xiao Y (2018) MicroRNA34a mediates atrial fibrillation through regulation of AnkyrinB expression. Molecular medicine reports 17: 84578465. [Crossref]

61. Zhao Y, Yuan Y, Qiu C (2016) Underexpression of CACNA1C Caused by Overexpression of microRNA-29a Underlies the Pathogenesis of Atrial Fibrillation. Med Sci Monit 22: 2175-2181. [Crossref]

62. Liu X, Zhang Y, Du W, Liang H, He H, et al. (2016) MiR-223-3p as a Novel MicroRNA Regulator of Expression of Voltage-Gated K+ Channel Kv4.2 in Acute Myocardial Infarction. Cell Physiol Biochem 39: 102-14. [Crossref]

63. Qiao G, Xia D, Cheng Z, Zhang G (2017) miR-132 in atrial fibrillation directly target connective tissue growth factor. Molecular medicine reports 16: 4143-4150. [Crossref]

64. Nassal DM, Wan X, Liu H, Maleski D, Ramirez-Navarro A, et al. (2017) KChIP2 is a core transcriptional regulator of cardiac excitability. eLife 6: e17304.

65. Mazurek SR, Calway T, Harmon C, Farrell P, Kim GH (2017) MicroRNA-130a Regulation of Desmocollin 2 in a Novel Model of Arrhythmogenic Cardiomyopathy. MicroRNA 6: 143-150. [Crossref]

66. Reilly SN, Liu X, Carnicer R, Recalde A, Muszkiewicz A, et al. (2016) Up-regulation of miR-31 in human atrial fibrillation begets the arrhythmia by depleting dystrophin and neuronal nitric oxide synthase. Sci Transl Med 8: 340ra74. [Crossref]

Copyright: ( 2018 Guzzolino E. This is an open-access article distributed under the terms of the Creative Commons Attribution License, which permits unrestricted use, distribution, and reproduction in any medium, provided the original author and source are credited. 